\title{
TRIANGULAR MATRIX ALGEBRAS OVER HENSEL RINGS
}

\author{
JOSEPH A. WEHLEN
}

\begin{abstract}
Let $(R, m)$ be a local Hensel ring and $A$ an algebra over $R$ which is finitely generated and projective as an $R$-module. If $A$ contains a complete set of mutually orthogonal primitive idempotents $e_{1}, \cdots, e_{n}$ indexed so that $e_{i} N e_{j} \subseteq m A$ whenever $i \geqq j$, we show that $A$ is isomorphic to a generalized triangular matrix algebra and that $A$ is the epimorphic image of a finitely generated, projective $R$-algebra $B$ of Hochschild dimension less than or equal to one.
\end{abstract}

Introduction. The class of residue algebras of semiprimary hereditary algebras has been thoroughly discussed in [5], [9], [2], [6] and [12]. They consist of those finitely generated algebras $A$ over a field $R$ which contain a complete set of mutually orthogonal primitive idempotents $e_{1}, \cdots, e_{n}$ which can be indexed so that $e_{i} N e_{j}=(0)$ whenever $i \geqq j$, where $N$ denotes the Jacobson radical of $A$, and $A / N$ is $R$-separable. All such algebras are isomorphic to generalized triangular matrix algebras.

The purpose of this paper is to show that every finitely generated, projective algebra $A$ over a local Hensel ring $(R, m)$ satisfies the "triangular" idempotent condition $e_{i} N e_{j} \subseteq m A$ whenever $i \geqq j$ if and only if $A$ is a residue algebra of a finitely generated, projective algebra $B$ of Hochschild dimension less than or equal to one. We will call such algebras "almost one-dimensional."

Such an algebra $B$, the maximal algebra for $A$, is usually neither semiprimary nor hereditary. In fact, if we assume that $R$ is also noetherian, it has been shown in [11] that $B$ is hereditary if and only if $R$ is a field ( $R$ $\operatorname{dim} B \leqq 1)$ or a DVR $(R-\operatorname{dim} B=0) ; B$ is semiprimary if and only if $R$ has a nilpotent radical-in which case every finitely generated, projective algebra over $R$ has infinite global dimension-or $R$ is a field.

A generalization of a result of $S$. U. Chase [2] will provide a sort of converse to Theorem 2.7 of [10] in the case of a Hensel ring:

Received by the editors October 5, 1971.

AMS (MOS) subject classifications (1970). Primary 16A32, 16A62; Secondary 13J15, 16A42, 16A46, 18H15.

Key words and phrases. Hochschild dimension, cohomological dimension, generalized triangular matrix algebra, Hensel ring, maximal algebra, lifting idempotents.

(c) American Mathematical Society 1973 
THEOREM 0. Let $A$ be a finitely generated, projective algebra over $a$ commutative ring $R$. If $R-\operatorname{dim} A=1$, then, for every ideal I containing $\boldsymbol{m} A$ or some maximal ideal $\boldsymbol{m}$ of $R, R-\operatorname{dim}(A / I)$ is finite.

Conventions. Throughout this paper, all rings will have one and all ring homomorphisms will take the identity to the identity. All modules are unitary. By a local ring $R$, we mean only that $R$ has a unique maximal ideal $\boldsymbol{m}$. By a finitely generated $R$-algebra or a projective $R$-algebra, we shall mean that $A$ is finitely generated or projective as a module over $R$. All homological dimensions will be taken as left modules and $R-\operatorname{dim} A=$ $\mathrm{hd}_{A^{e}}(A)$ will denote the Hochschild (or cohomological) dimension of the algebra $A$, where $A^{e}=A \otimes_{R} A^{\mathrm{op}}$. $N$ will always denote the Jacobson radical of the algebra $A$.

1. Almost one-dimensional algebras. We shall say that a finitely generated, projective algebra $A$ over a local Hensel ring $(R, m)$ is almost one-dimensional if every complete set of mutually orthogonal primitive idempotents $e_{1}, \cdots, e_{n}$ can be indexed so that $e_{i} N e_{j} \subseteq m A$ whenever $i \geqq j$. The definition is justified by the following results.

THEOREM 1. Let $A$ be a finitely generated, projective algebra over a local Hensel ring $R$. (a) If $R-\operatorname{dim} A \leqq 1$, then $A$ is almost one-dimensional. (b) If $A$ is almost one-dimensional, then $A / m A$ is triangular in the sense of Chase; i.e., A/mA contains a complete set of mutually orthogonal primitive idempotents $e_{1}, \cdots, e_{n}$ indexed so that $e_{i}(N / m A) e_{j}=(0)$ whenever $i \geqq j$. (c) If $A$ is almost one-dimensional, then $R-\operatorname{dim} A$ is finite.

(We note that the hypotheses that $R$ is Hensel and $A$ is $R$-projective are not necessary to prove (a) or (b).)

Proof. (a) is contained in Theorems 3.4 and 3.6 of [10]. (b) is obvious. For (c), an application of (b) and Theorem 4.1 of [2] shows that $R / \boldsymbol{m}-\operatorname{dim} A / \boldsymbol{m} A=\mathrm{gl} \operatorname{dim} A / \boldsymbol{m} A$ is finite. But then, by 2.1 of [10], $R$-dim $A=R / m-\operatorname{dim} A / \boldsymbol{m} A$ is finite.

By means of part (b) of Theorem 1 and the idempotent lifting theorem of G. Azumaya [1, Theorem 24], it is easy to deduce the following equivalent conditions for an algebra to be almost one-dimensional from Theorem 4.1 of [2].

THEOREM 2. If $A$ is a finitely generated, projective algebra over a local Hensel ring $R$, the following conditions are equivalent:

(a) $A$ is almost one-dimensional.

(b) There exists a complete set of mutually orthogonal primitive idempotents $e_{1}, \cdots, e_{n}$ of $A$ which can be indexed so that $e_{i} N e_{j} \subseteq m A$ whenever $i \geqq j$. 
(c) For every ideal I of $A$ containing $\boldsymbol{m} A, A / I$ has finite Hochschild dimension.

(d) $A /\left(N^{2}+m A\right)$ has finite Hochschild dimension.

Results of [2], [5] and [9] on the nilpotence degree of the Jacobson radical are translatable in terms of the nilpotence degree of $N$ modulo $\boldsymbol{m} A$ by use of the same techniques.

2. Construction of the maximal algebra. If $A$ is almost one-dimensional, we shall call the one-dimensional algebra $B$ which we are going to construct the "maximal algebra" for $A$.

Recall that a generalized triangular matrix algebra $T_{n}\left(A_{i} ; M_{i j} / R\right)$ is the algebra defined in the following way: the $A_{i}$ are algebras over $R$, the $M_{i j}$ are left $A_{i^{-}}$and right $A_{j}$-bimodules with $M_{i j}=(0)$ for $i>j$ and $M_{i i}=A_{i} ; R$ commutes with the $M_{i j}$. Multiplication is defined via homomorphisms $\phi_{i j}^{t}: M_{i t} \otimes_{A_{t}} M_{t j} \rightarrow M_{i j}$ with the $\phi_{i t}^{t}$ and the $\phi_{i t}^{i}$ isomorphisms; these mappings satisfy the "associative" law: $\phi_{i k}^{j}\left(\mathrm{id}_{i j} \otimes \phi_{j k}^{t}\right)=\phi_{i k}^{t}\left(\phi_{i t}^{j} \otimes \mathrm{id}_{t k}\right)$. These functions induce a matrix multiplication on

$$
T_{n}\left(A_{i}: M_{i j} / R\right)=\left\{\left(\begin{array}{cccc}
a_{11} & m_{12} & \ldots & m_{1 n} \\
& a_{22} & \ldots & m_{2 n} \\
& & : &
\end{array}\right): a_{i i} \text { in } A_{i}, m_{i j} \text { in } M_{i j}\right\}
$$

(cf. [10] or [6, p. 465]).

THEOREM 3. Let $A$ be a finitely generated, projective algebra over a local Hensel ring $R$. If $A$ is almost one-dimensional, then $A$ is isomorphic to a generalized triangular matrix algebra over $R$.

Proof. The proof is essentially contained in the case where $A / N$ is isomorphic to a direct sum of division algebras over $R$. In this case, the $e_{i}$ are all nonisomorphic. Now $e_{i} A e_{i}$ is a separable, projective $R$-algebra. So $S=\sum_{i=1}^{n} e_{i} A e_{i}$ is the inertial subalgebra whose existence is guaranteed by [10, Proposition 2.5] and [1, Theorem 33], since $S / m S=A / N$.

Furthermore, if $j>k$, then

$$
e_{j} A e_{k}=e_{j} S e_{k}+e_{j} N e_{k} \subseteq \boldsymbol{m}\left(\oplus \sum_{i, t} e_{i} A e_{t}\right),
$$

where the first sum is not direct. Thus $e_{j} A e_{k}=\boldsymbol{m}\left(e_{j} A e_{k}\right)$. Hence by Nakayama's lemma, $e_{j} A e_{k}=(0)$ for all $j>k$. Hence under the natural maps induced by the multiplication in $A$ from $e_{i} A e_{t}$ and $e_{t} A e_{j}$ to $e_{i} A e_{j}$, we have that $A \cong T_{n}\left(e_{i} A e_{i} ; e_{i} A e_{j} / R\right)$.

The general case follows by noting that if $e=\sum_{i=1}^{n} e_{i 1}$, where $i$ denotes the distinct isomorphism classes of primitive idempotents, then by 
[6, Proposition 2]: $R / \boldsymbol{m}-\operatorname{dim} e(A / \boldsymbol{m} A) e=\mathrm{gl} \operatorname{dim} e(A / \boldsymbol{m} A) e=\mathrm{gl} \operatorname{dim} A / \boldsymbol{m} A=$ $R / m-\operatorname{dim} A / m A$. Again, by Theorem 2.1 of [10], $R-\operatorname{dim} A=R-\operatorname{dim} e A e . e A e$ is almost one-dimensional and $e A e / e N e$ is isomorphic to a direct sum of division algebras. Finally, following the techniques of [10] and [6], we have that if $e A e \cong T_{n}\left(e_{i 1} A e_{i 1} ; e_{i 1} A e_{j 1} / R\right)$, then $A \cong T_{n}\left(A_{i} ; M_{i j} / R\right)$ where $A_{i}=\left(e_{i 1} A e_{i 1}\right)_{s_{i} \times s_{i}}$ and $M_{i j}=\left(e_{i 1} A e_{j 1}\right)_{s_{i} \times s_{j}}$, the $s_{i}$ by $s_{j}$ matrices with entries from $e_{i 1} A e_{j 1}$.

We are now in a position to construct the maximal algebra for $A$. Let $N^{*}=\sum_{j<k} M_{j k}, P=\sum_{i=1}^{n-1} M_{i, i+1}$, and $M=\sum_{j+1<k} M_{j k}$. Clearly, $A=S \oplus N^{*}=$ $S \oplus P \oplus M$ as $S$-S bimodules, where $P, M$, and $N^{*}$ are finitely generated, projective $R$-modules. Set $P^{(k)}$ equal to the $k$-fold tensor product of $P$ with itself over $S$. The middle-four-interchange gives that $\left(P \otimes_{S} P\right) \otimes R / m \cong$ $P / m P \otimes_{S / m S} P / m P$. An easy induction then shows that $P^{(k)} / m P^{(k)} \cong$ $(P / m P)^{(k)}$.

Let $B=S \oplus P \oplus P^{(2)} \oplus P^{(3)} \oplus \cdots=S \oplus P \oplus T$ be the algebra with multiplication defined as in a graded ring with $S=P^{(0)}$; i.e.,

$$
\left(p_{1} \otimes \cdots \otimes p_{q}\right) \cdot\left(p_{1}^{\prime} \otimes \cdots \otimes p_{s}^{\prime}\right)=\left(p_{1} \otimes \cdots \otimes p_{q} \otimes p_{1}^{\prime} \otimes \cdots \otimes p_{s}^{\prime}\right)
$$

(cf. [8, Definition 1.4]). To apply Theorem 2.1 of [10], we need to know that $T$ is finitely generated and projective as an $R$-module. That $T$ is $R$-projective follows by noting that $P$ is $R$-projective and hence by [3, Proposition 2.3] $S$-projective; whence $P \otimes_{S} P$ is $S$-projective and therefore $R$-projective. That $T$ is finitely generated is shown by the following: Since $P / m P$ is the $S / m S$-complement of $(N / m A)^{2}$ in $(N / m A)$, by $[9$, p. 71$],(P / m P)^{(n)}=(0)$ for some $n$; so by Nakayama's lemma, $P^{(n)}=(0)$. Finite generation is now clear.

But $B / \boldsymbol{m} B$ is the maximal algebra over the triangular algebra $A / \boldsymbol{m} A$. Hence, $R-\operatorname{dim} B \leqq 1$. Defining $f: B \rightarrow A$ by $f\left(s, p, p_{1} \otimes p_{2}, \cdots\right)=s+p+$ $p_{1} p_{2}+\cdots$, we obtain an algebra epimorphism of $B$ onto $A$. It is clear that $B / T$ is isomorphic to $A / N^{*^{2}}$. Thus we have just shown

THEOREM 4. Let $A$ be a finitely generated, projective algebra over a local Hensel ring $R$. The following are equivalent.

(a) $A$ is almost one-dimensional.

(b) There is a finitely generated, projective algebra $B$ over $R$ such that $R-\operatorname{dim} B \leqq 1, A$ is an epimorphic image of $B$ with $B / T \cong A / N^{*^{2}}$, where $N^{*^{2}}$ and $T$ are the squares of the $R$-complements of the inertial subalgebras of $A$ and $B$ respectively.

Combining Theorems 2 and 4, one obtains the complete analogue of the results of [9], [2] and [6] for finitely generated triangular algebras over a field. 
3. Miscellaneous results and corollaries. In [4, p. 311] S. Eilenberg gave necessary and sufficient conditions for a finitely generated algebra over a field to have a given Hochschild dimension. This characterization involved the Jacobson radical of the algebra. We note the following extension of that result: (Recall that an inertial subalgebra of an algebra $A$ is a separable subalgebra $S$ of $A$ such that $A=S+N$, where the sum is not necessarily direct.)

THEOREM 5. Let A be a finitely generated, projective algebra over a local ring $R$. Suppose that $A$ has finite Hochschild dimension. Let $S$ be an inertial subalgebra of $A$ such that $A=S \oplus I$ is an $R$-direct sum for some ideal I of $A$. Then the following hold: (a) $R-\operatorname{dim} A=\mathrm{hd}_{A}(S)$; and (b) $R-\operatorname{dim} A=$ $1+\operatorname{hd}_{A}(I)$.

Proof. Since $A$ and $S$ are $R$-projective, by the argument of Theorem 2.1 of [10], and by the corollary to Theorem 3 of [4], one sees that the following equalities hold:

$$
\begin{aligned}
R-\operatorname{dim} A & =R / \boldsymbol{m}-\operatorname{dim} A / \boldsymbol{m} A=\operatorname{hd}_{A / m A}(A / N) \\
& =\operatorname{hd}_{A / m A}(S / m S)=\operatorname{hd}_{A}(S) .
\end{aligned}
$$

The second part follows directly from the first.

Corollary 5.1. Let the setting be as in the theorem. Then $A$ is onedimensional if and only if $I$ is projective as an $A$-module; $A$ is $R$-separable if and only if $I=(0)$.

In particular, the setting of Theorem 5 always holds true for the algebras considered in this paper. It gives us a particularly interesting characterization of which almost one-dimensional algebras are actually one-dimensional:

COROllary 5.2. Suppose $A$ is a finitely generated, projective, almost one-dimensional algebra over a local Hensel ring $R$. Then, $A=S \oplus N^{*}$, where $N^{*}$ is an ideal of $A$, and $\mathrm{hd}_{A}(S)=R-\operatorname{dim} A$. Moreover, $A$ has Hochschild dimension one if and only if $N^{*}$ is projective as an $A$-module.

Finally, one can give the following characterization of almost onedimensional algebras based upon the ideal $N^{*}$ rather than the Jacobson radical, which allows one to restate Theorem 2 in terms of $N^{*}$.

THEOREM 6. Let $A$ be a finitely generated, projective algebra over a local Hensel ring $R$; let $A$ have finite Hochschild dimension. $A$ is almost one-dimensional if and only if there is an ideal $N^{*}$ of $A$ such that $A=S \oplus N^{*}$ (direct sum as $R$-modules), where $S$ is the inertial subalgebra of $A$, and there exists a complete set of mutually orthogonal primitive idempotents $e_{1}, \cdots, e_{n}$ such that $e_{i} N^{*} e_{j}=(0)$ for $i \geqq j$. 
Proof. The "only if" part is a direct consequence of Theorem 3. Suppose that $A=S \oplus N^{*}$, and $A$ contains the required set of idempotents. Then $e_{i} N e_{j}=e_{i}\left(m S+N^{*}\right) e_{j}=e_{i}(m S) e_{j} \subseteq m A$ whenever $i \geqq j$ and $N$ denotes the Jacobson radical of $A$.

CONCLUDING Remarks. The algebras of Hochschild dimension one over a local Hensel ring act as the semiprimary hereditary algebras over a field. In fact, an almost one-dimensional algebra $A$ and its maximal algebra $B$ can easily be seen to satisfy the definition of quasi-cyclic algebras and related algebras (with $N^{*}$ and $T$ replacing the Jacobson radicals) as given by Hochschild in [7, pp. 369 and 372].

It would be useful to know that the ideal $N^{*}$ was a radical of the algebra $A$. In a future paper, we will show that if $R$ is a noetherian domain which is a local Hensel ring, then $N^{*}$ is the (Baer) lower radical of $A$. This is not true if $R$ is a complete local ring with nilpotent radical, in which case the Jacobson and lower radicals are equal.

\section{BIBLIOGRAPHY}

1. G. Azumaya, On maximally central algebras, Nagoya Math. J. 2 (1951), 119-150. MR 12, 669.

2. S. U. Chase, A generalization of the ring of triangular matrices, Nagoya Math. J. 18 (1961), 13-25. MR 23 \#A919.

3. F. Demeyer and E. Ingraham, Separable algebras over commutative rings, Lecture Notes in Math., vol. 181, Springer-Verlag, Berlin and New York, 1971. MR 43 \#6199.

4. S. Eilenberg, Algebras of cohomologically finite dimension, Comment. Math. Helv. 28 (1954), 310-319. MR 16, 442.

5. S. Eilenberg, H. Nagao and T. Nakayama, On the dimension of modules and algebras. IV. Dimension of residue rings of hereditary rings, Nagoya Math. J. 10 (1956), 87-95. MR 18, 9.

6. M. Harada, Hereditary semi-primary rings and tri-angular matrix rings, Nagoya Math. J. 27 (1966), 463-484. MR 34 \#4300.

7. G. Hochschild, On the structure of algebras with nonzero radical, Bull. Amer. Math. Soc. 53 (1947), 369-377. MR 8, 561.

8. J. Jans, On segregated rings and algebras, Nagoya Math. J. 11 (1957), 1-7. MR $19,249$.

9. J. P. Jans and T. Nakayama, On the dimension of modules and algebras. VII. Algebras with finite-dimensional residue-algebras, Nagoya Math. J. 11 (1957), 67-76. MR 19, 250.

10. J. A. Wehlen, Algebras of finite cohomological dimension, Nagoya Math. J. 43 (1971), 127-135.

11. Cohomological dimension and global dimension of algebras, Proc. Amer. Math. Soc. 32 (1972), 75-80.

12. A. Zaks, Semiprimary rings of generalized triangular type, J. Algebra 9 (1968), 54-78. MR 36 \#6455.

Department of Mathematics, Ohio University, Athens, OHio 45701 